\title{
Effect of melt cooling rate on glass transition kinetics and structural relaxation of Vit1 metallic glass
}

\author{
Wei Zhang, Qing-chun Xiang, *Ying-dong Qu, Qing-feng Li, Ying-lei Ren, Ke-qiang Qiu \\ School of Materials Science and Engineering, Shenyang University of Technology, Shenyang 110870, China
}

\begin{abstract}
The thin ribbons and the bulk cylindrical rods with diameters of $2 \mathrm{~mm}$ and $10 \mathrm{~mm}$ of the Vit1 metallic glass (MG) were prepared by the single roller melt spinning method and the copper mold injection casting method, respectively. The cooling rates of the samples during melt solidification were evaluated. The glass transition behaviors of three groups of MG samples with different solidification cooling rates were studied by differential scanning calorimetry (DSC) at different heating rates. The effects of melt cooling rate on the glass transition kinetic parameters such as apparent activation energy $(E)$ and fragility parameter $(m)$ of the Vit1 MG were studied using the Kissinger and the Vogel-Fulcher-Tammann (VFT) equations. Additionally, the structural relaxation enthalpy $\left(\Delta H_{\text {rel }}\right)$ of three groups of MG samples was quantitatively analyzed by DSC through multi-step temperature rise and fall measurements. Results show that the melt cooling rate $(R)$ has a significant effect on the glass transition kinetics and the structural relaxation of the Vit1 MG. As $R$ decreases in the order of magnitude, the glass transition temperature $\left(T_{\mathrm{g}}\right), E, m$, and $\Delta H_{\text {rel }}$ of the Vit1 MG gradually decreases. Furthermore, in the range of the experimental cooling rates, $E, m$, and $\Delta H_{\text {rel }}$ all have an approximately linear relationship with $\lg R$.
\end{abstract}

Key words: glass transition; structural relaxation; apparent activation energy; fragility parameter; cooling rate; Vit1 metallic glass
CLC numbers: TG244 ${ }^{+} .1$
Document code: A
Article ID: 1672-6421(2021)02-118-06

\section{Introduction}

The formation of metallic glasses (MGs) is considered to be the result of "freezing" the translational motion of a large range of atoms in the melt during rapid cooling ${ }^{[1]}$. It is generally believed that during the cooling process, the viscosity of the alloy melt will increase rapidly as the degree of undercooling increases. When the cooling rate is low, the degree of undercooling and the viscosity of the melt are relatively small, and the internal structure of the melt will be basically kept in equilibrium due to the rapid movement of atoms, and finally, the melt will solidify in the form of crystals. Only when the cooling rate is sufficiently high to a certain extent, that is, the degree of undercooling and the viscosity of the melt are high enough (the viscosity reaches $10^{12} \mathrm{~Pa} \cdot \mathrm{s}{ }^{[2]}$, the movement of atoms in the melt becomes very slow, and the time required for its structure to transit to the

\footnotetext{
*Ying-dong Qu
}

Male, born in 1975, Professor. His research interests mainly focus on carbon fiber reinforced aluminum matrix composites, high entropy alloy, and casting new materials.

E-mail: quydong@sut.edu.cn;

Received: 2020-09-21; Accepted: 2020-12-10 internal equilibrium state by relaxation is much longer than the experimental time scale), the melt can undergo glass transition due to its loss of equilibrium, and form a glassy solid phase ${ }^{[1,3]}$. It is not difficult to understand that the cooling rate of the alloy melt during its vitrification process directly determines the time for the undercooled melt to undergo structural relaxation, thus affecting the local atomic structure in the MG and then affecting the kinetic and thermodynamic stability ${ }^{[4,5]}$ as well as the performances of the MG.

Many researchers have studied the effects of the cooling rate of glass-forming alloy liquid on the glass transition kinetics and structural relaxation ${ }^{[6-8]}$. However, the cooling experiments are usually performed from the supercooled liquid region of alloys. In addition, the experiments are usually performed at the cooling rate $(R)$ of less than $10 \mathrm{~K} \cdot \mathrm{s}^{-1}$, and the $R$ spans only one or two orders of magnitude. In fact, MGs are solidified from higher temperature melts at higher cooling rates, which is significantly different from the traditional analysis experiments. Therefore, it is interesting to evaluate the change trends of the glass transition kinetics and structural relaxation that are caused by the different rapid cooling histories of the solidification processes 
for MGs. In this work, taking the Vit1 MG, a well-known MG developed by Peker et al ${ }^{[9]}$, to serve our purpose, the influences of melt cooling rate during the solidification process on the glass transition kinetics and structural relaxation of the Vit1 MG were quantitatively analyzed. The work can provide a basis for the theoretical exploration on the glass transition of alloy melts.

\section{Experimental procedure}

Master ingots with the nominal composition of $\mathrm{Zr}_{41.2} \mathrm{Ti}_{13.8} \mathrm{Ni}_{10.0} \mathrm{Cu}_{12.5} \mathrm{Be}_{22.5}$ (Vit1 alloy, atomic percentage) were prepared by the vacuum induction melting of a mixture of $\mathrm{Zr}, \mathrm{Ti}, \mathrm{Ni}, \mathrm{Cu}$ and $\mathrm{Be}$ raw materials with a purity higher than $99.9 \%$ in high purity argon atmosphere. Next, the master alloy ingots were cut into small pieces and placed in quartz tubes with nozzles of different shapes and sizes. Then, the ingots were remelted in the induction furnace. The thin ribbons with a thickness of about $50 \mu \mathrm{m}$ and the rod-shaped samples with diameters $(D)$ of $2 \mathrm{~mm}$ and $10 \mathrm{~mm}$ were cast by the single copper roller melt spinning method and the copper mold injection method, respectively.

TA DSC Q-100 differential scanning calorimeter (DSC) was used to analyze the glass transition kinetic behaviors of the samples, and the heating rates were 5, 10, 20 and $40 \mathrm{~K} \cdot \mathrm{min}^{-1}$, respectively. In addition, the structural relaxation enthalpy $\left(\Delta H_{\text {rel }}\right)$ of all the samples was analyzed by DSC through the multi-step heating and cooling measurements. The detailed experimental process was as follows: firstly, the DSC sample was heated to the supercooled liquid phase region at a heating rate of 3 $\mathrm{K} \cdot \mathrm{min}^{-1}$, then the sample was cooled to room temperature at the same cooling rate $\left(3 \mathrm{~K} \cdot \mathrm{min}^{-1}\right)$, and subsequently, the sample was heated to the supercooled liquid phase region at the same heating rate $\left(3 \mathrm{~K} \cdot \mathrm{min}^{-1}\right)$ again. During the two heating processes, the curves of the heat flow rate of the sample were recorded.

\section{Results}

\subsection{Cooling rates of samples during preparation}

In this work, the cooling rate $(R)$ of the MG ribbons prepared by the single roller melt spinning method is in the order of $10^{5}-10^{6} \mathrm{~K} \cdot \mathrm{s}^{-1}{ }^{[10]}$. Nevertheless, the $R$ of the bulk samples prepared by the copper mold injection method is about $4,000 / D^{2}\left(\mathrm{~K} \cdot \mathrm{s}^{-1}\right)^{[1]}$, where $D$ is the diameter of the sample, that is, the $R$ of the copper mold injection samples with the diameters of $2 \mathrm{~mm}$ and $10 \mathrm{~mm}$ is about $1,000 \mathrm{~K} \cdot \mathrm{s}^{-1}$ and $40 \mathrm{~K} \cdot \mathrm{s}^{-1}$, respectively. The $R$ of the three groups of experimental samples are all greater than the critical cooling rate $\left(R_{\mathrm{c}}\right)$ of the Vit1 $\mathrm{MG}\left(R_{\mathrm{c}}<10 \mathrm{~K} \cdot \mathrm{s}^{-1}\right)^{[9]}$, which further verifies that all the samples have an amorphous structure.

\subsection{Thermodynamic curves and apparent activation energy of glass transition of samples}

Figure 1 shows the DSC curves around the glass transition temperature $\left(T_{\mathrm{g}}\right)$ of three groups of Vit1 MG samples heated at different heating rates. Table 1 shows the $T_{\mathrm{g}}$ values of three groups of Vit1 amorphous samples at different heating rates. It can be seen that the $T_{\mathrm{g}}$ of all the samples increases with the increase of heating rate, showing the obvious kinetic characteristics, furthermore, the endothermic behavior of the samples during the glass transition becomes more and more obvious with the increase of heating rate.
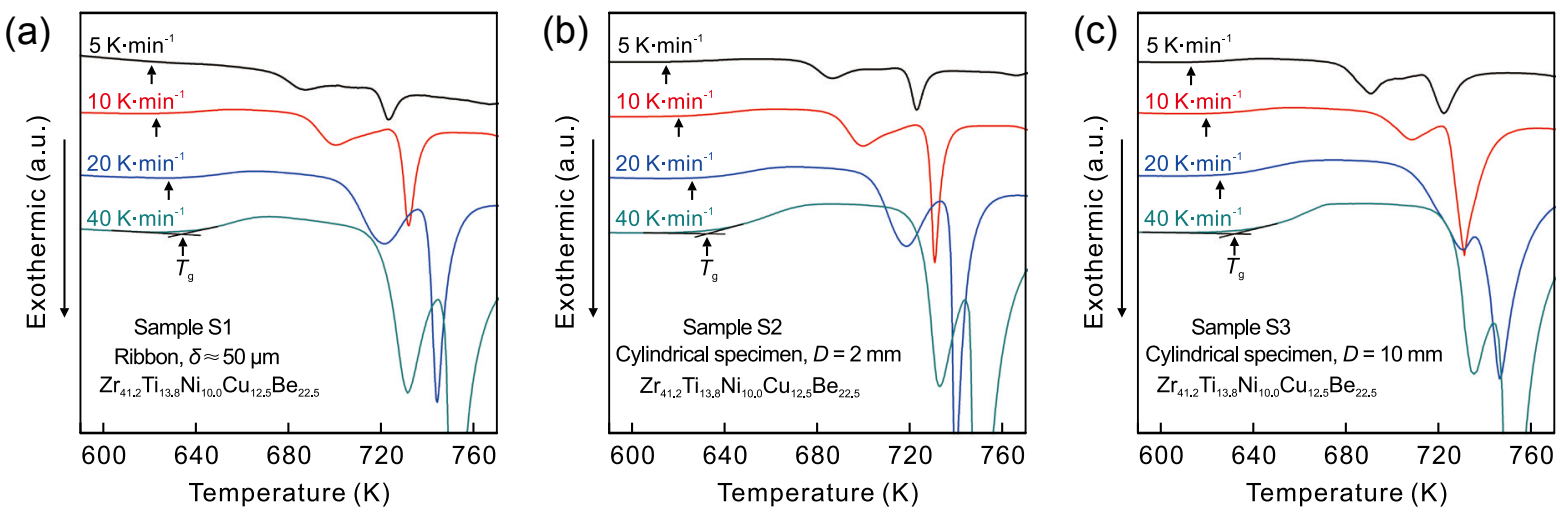

Fig. 1: DSC curves of three groups of Vit1 MG samples at different heating rates

Table 1: Preparation characteristics of three groups of Vit1 MG samples and their $T_{\mathrm{g}}$ values at different heating rates

$\begin{array}{ccccccc}\text { Sample No. } & \begin{array}{c}\text { Sample size } \\ \text { specification }\end{array} & \begin{array}{c}\text { Cooling rate, } R \\ \left(\mathbf{K} \cdot \mathbf{s}^{-1}\right)\end{array} & \begin{array}{c}\text { Glass transition temperature, } T_{\mathrm{g}}(\mathbf{K}) \\ \mathbf{5 ~ K} \cdot \mathbf{m i n}^{-1}\end{array} & \mathbf{1 0} \mathbf{~ K} \cdot \mathbf{m i n}^{-1} & \mathbf{2 0} \mathbf{~ K} \cdot \mathbf{m i n}^{-1} & \mathbf{4 0} \mathbf{~ K} \cdot \mathrm{min}^{-1} \\ \text { S1 } & \delta \approx 50 \mu \mathrm{m} & 10^{5}-10^{6} & 618.7 & 622.9 & 627.9 & 633.0 \\ \text { S2 } & D=2 \mathrm{~mm} & 1000 & 614.8 & 619.9 & 625.8 & 631.7 \\ \text { S3 } & D=10 \mathrm{~mm} & 40 & 613.5 & 619.0 & 625.1 & 631.3\end{array}$


It can also be seen from Table 1 that the $T_{\mathrm{g}}$ value of the MG sample with the smaller sample size will increase slightly at the same heating rate. This phenomenon is consistent with the most experimental results and theoretical viewpoints. That is, the faster the cooling rate, the higher the temperature point required for "freezing" the movement of the atoms and forming the vitrification during the solidification of the melt, and the easier the alloy system to achieve the non-equilibrium transition, meanwhile, the higher the $T_{\mathrm{g}}$ of the $\mathrm{MG}^{[12]}$.

The glass transition of the MG during the heating process is the result of the transformation of the atomic structure in the alloy to the equilibrium supercooled liquid structure due to the diffusion and rearrangement of atoms. In this process, the atoms absorb energy to overcome the energy barrier (activation energy) required for the alloy transiting from a glassy state to a supercooled liquid state, and it will appear as an endothermic reaction on the thermodynamic curve. In this work, in order to reflect the influence of the melt cooling rate on the structural characteristics of the Vit1 MG, the apparent activation energy for the glass transition of the three groups of samples was calculated using the Kissinger method. The formula is ${ }^{[13]}$.

$$
\ln \left(T^{2} / q\right)=E / R_{\mathrm{gas}} T+C
$$

where $q$ is the heating rate; $E$ is the apparent activation energy; $R_{\text {gas }}$ is the ideal gas constant; $T$ is the characteristic temperature (here referred to as the glass transition temperature $T_{\mathrm{g}}$ ); $C$ is the constant. Based on the data in Table 1, the $\ln \left(T^{2} / q\right)-1 / T$ plot map of the three groups of Vit1 MG samples is drawn, as shown in Fig. 2. The results show that the $\ln \left(T^{2} / q\right)$ of each group of samples has a good linear relationship with $1 / T$. Therefore, the experimental data can be fitted with linear regression according to the Kissinger equation (1), and the apparent activation energy $E$ of each MG sample can be calculated according to the slope of the fitted line. It is calculated that the apparent activation energy of the Vit1 MG ribbon (Sample S1), of which the melt cooling rate is on the order of $10^{5}-10^{6} \mathrm{~K} \cdot \mathrm{s}^{-1}$, is $454.77 \mathrm{~kJ} \cdot \mathrm{mol}^{-1}$, which is close to that of the atomized Vit $1 \mathrm{MG}$ particles prepared by $\mathrm{Li}$ et al. ${ }^{[14]}$ at a cooling rate of $10^{5} \mathrm{~K} \cdot \mathrm{s}^{-1}(E=435.50$ $\left.\mathrm{kJ} \cdot \mathrm{mol}^{-1}\right)$. The apparent activation energies of Samples S2 and $\mathrm{S} 3$ are $384.69 \mathrm{~kJ} \cdot \mathrm{mol}^{-1}$ and $364.68 \mathrm{~kJ} \cdot \mathrm{mol}^{-1}$, respectively. The

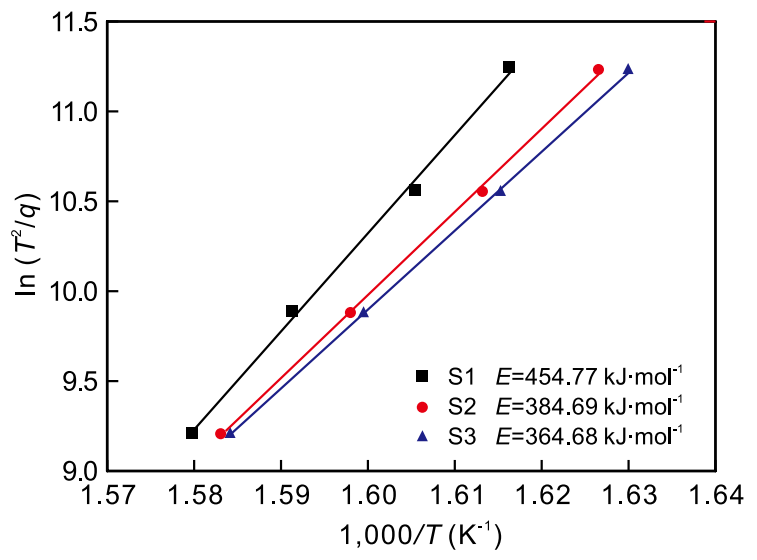

Fig. 2: Kissinger fitting curves of apparent activation energy for glass transition of three groups of Vit1 MG samples calculation results show that as the cooling rate of the alloy melt decreases, the apparent activation energy for the glass transition of the Vit1 MG sample gradually decreases. This phenomenon is consistent with the research results of the Cobased MGs presented by Yuan et al ${ }^{[15]}$.

\subsection{VFT equation and fragility parameter of samples}

During the heating processes at different heating rates, the kinetic characteristics of the glass transition of MGs are closely related to the change of the structural relaxation time. Masuhr et al. ${ }^{[16]}$ found that the relationship between the structural relaxation time and temperature at the glass transition of MGs conforms to the Vogel-Fulcher-Tammann (VFT) equation. This also shows that the kinetic characteristics, of which the glass transition of MGs varies with the heating rate, can effectively reflect the fragility of the alloy systems. In order to study the glass transition kinetics of the three groups of Vit1 MG samples at different heating rates, the correlation between $T_{\mathrm{g}}$ and $q$ is fitted by the VFT equation ${ }^{[17]}$ :

$$
\ln q=\ln B-\frac{D^{*} T_{0}}{T_{\mathrm{g}}-T_{0}}
$$

where $B$ is the constant; $D^{*}$ is the strength index; $T_{0}$ is the VFT characteristic temperature. By fitting the experimental data of the three groups of MG samples in Table 1 with Eq. (2), the $\ln q$ vs $T_{\mathrm{g}}$ curves can be obtained, as shown in Fig. 3. It can be found that all the three groups of MG samples can well meet the VFT nonlinear fitting equation. The fitting parameters $\ln B$, $D^{*}$ and $T_{0}$ of the VFT equation for the three groups of MG samples are listed in Table 2 . The glassy fragility parameter ( $m$ ) corresponding to the $T_{\mathrm{g}}$ points of the three groups of MG samples at the specific heating rates can be calculated with the VFT fitting parameters as follows ${ }^{[12]}$ :

$$
m=\frac{D^{*} T_{0} T_{\mathrm{g}}}{\left(T_{\mathrm{g}}-T_{0}\right)^{2} \ln 10}
$$

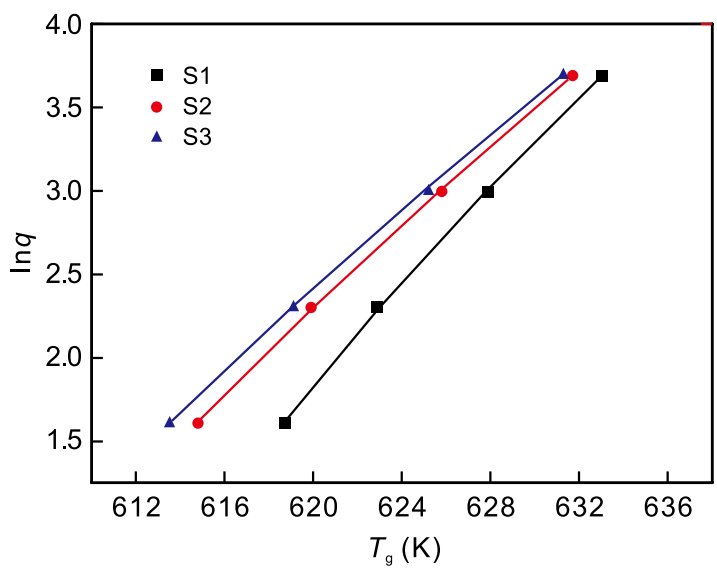

Fig. 3: Experiment results of Inq vs $T_{\mathrm{g}}$ and fitting curves according to VFT equation for three groups of Vit1 MG samples 
According to Eq. (3), the $T_{\mathrm{g}}$ at the heating rate of $20 \mathrm{~K} \cdot \mathrm{min}^{-1}$ was selected to calculate the $m$ values of three groups of Vit1 MG samples, and their results are also listed in Table 2. It can be seen that their $m$ values are in the range of 30-38, which is close to the range of 34-39 for a series of Zr-based MGs calculated by Perera ${ }^{[18]}$ using the same method. According to the classification of liquid fragility proposed by Angell ${ }^{[19]}$, the $m$ value of strong liquid is generally less than 30 , and that of fragile glass forming liquid is usually greater than 100 . For alloy systems with glass forming ability, $m$ values are usually between 25 and 100. Generally, MGs with $m<35$ are considered to be strong, and those with $m>35$ are fragile MGs ${ }^{[12]}$. For the Vit1 MG samples in this work, the $m$ values are close to that of the strong MGs. In addition, the comparison of the calculation results shows that as the cooling rate of the alloy melt decreases, the fragility parameter of the Vit1 MG samples has a decreasing trend.

Table 2: Fitting parameters $\operatorname{In} B, D^{*}$ and $T_{0}$ of VFT equation and fragility parameter $m$ of three groups of MG samples

$\begin{array}{cccccc}\text { Sample No. } & \ln B & D^{*} & T_{0}(\mathrm{~K}) & T_{\mathrm{g}}\left(\mathbf{q}=\mathbf{2 0} \mathrm{K} \cdot \mathrm{min}^{-1}\right) & \mathbf{m} \\ \text { S1 } & 17.06 & 2.71 & 526.22 & 627.9 & 37.45 \\ \text { S2 } & 21.82 & 6.42 & 466.59 & 625.8 & 32.11 \\ \text { S3 } & 24.14 & 8.91 & 439.66 & 625.1 & 30.86\end{array}$

\subsection{Structural relaxation enthalpy of samples}

MGs are in a thermodynamically metastable state. Under heating conditions controlled in a certain temperature range or even under normal temperature and pressure conditions, they have a tendency to transform to an equilibrium crystalline state. Their structure will change on the atomic scale, that is, they will change to another metastable glassy state with lower energy. This process is called structural relaxation. For MGs with the same composition, a different thermal history of melt cooling will result in obvious differences in atomic configurations and thermodynamic states, however, the value of the structural relaxation enthalpy can give the most intuitive reflection of these differences. In this work, in order to analyze the influence of melt cooling rate on the structural characteristics and thermodynamic state of Vit1 MG, the structural relaxation enthalpies of the three groups of samples were attained using DSC through multi-step heating and cooling measurements. The specific method is described in Refs. [20, 21].

Taking Sample S2 as an example, firstly, the as-cast MG sample was heated from room temperature to the supercooled liquid phase region by DSC at the heating rate of $3 \mathrm{~K} \cdot \mathrm{min}^{-1}$ (Run 1). The endpoint temperature of heating selected in this work is $650 \mathrm{~K}$, which is higher than the $T_{\mathrm{g}}$ but lower than the crystallization temperature. Under this heating condition, after the sample reaches the temperature in the supercooled liquid phase region, there is sufficient time for the structural relaxation to occur in the sample, and at the same time, the crystallization can be avoided. After the sample rose to the specified endpoint temperature, immediately it was cooled to room temperature at the same rate of $3 \mathrm{~K} \cdot \mathrm{min}^{-1}$. Then, the relaxed sample was heated again to the supercooled liquid phase region at the same heating rate (Run 2). During the twostep heating process, the curves of heat flow rates are plotted as shown in Fig. 4, where $W_{\text {ini }}$ is the heat flow rate (Run 1) of the initial sample, and the $W_{\text {rel }}$ is the heat flow rate (Run 2) of the relaxed sample. Since the experiment was carried out with the same sample at the same rate of heating and cooling, there

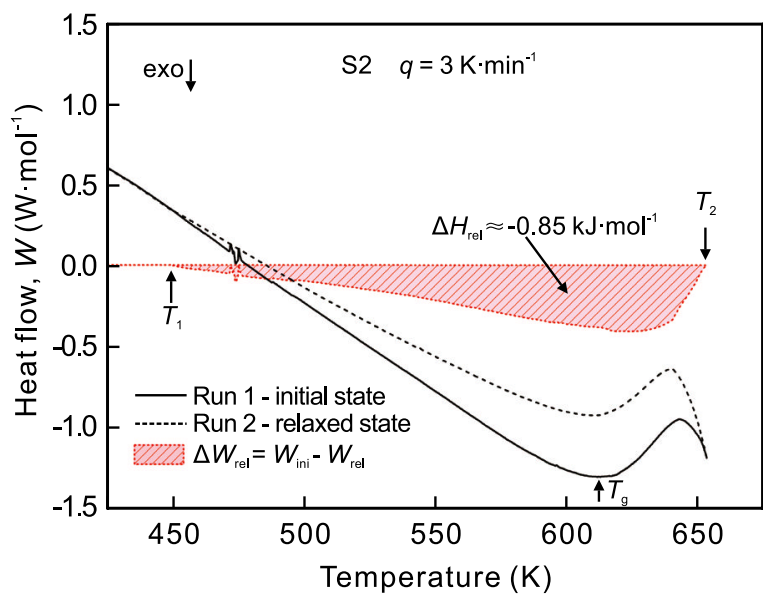

Fig. 4: Curves of heat flow rate of Vit1 MG Sample S2 before and after structural relaxation and calculation of structural relaxation enthalpy

is no need to use the baseline to calibrate the heat flow rate curves. The structural relaxation enthalpy $\Delta H_{\text {rel }}$ of the sample can be directly calculated by the following formula ${ }^{[20]}$.

$$
\Delta H_{\mathrm{rel}}=q^{-1} \int_{T_{1}}^{T_{2}} \Delta W_{\mathrm{rel}}(T) \mathrm{d} T
$$

where $\Delta W_{\text {rel }}$ is the difference of the heat flow rates between the two times of heating, $\Delta W_{\text {rel }}=W_{\text {ini }}-W_{\text {rel }} ; T_{1}$ and $T_{2}$ are the temperature points at which the heat flow rates of the as-cast and relaxed samples begin to differ before the glass transition temperature and coincide again after the glass transition temperature, respectively.

Through calculation, the $\Delta H_{\text {rel }}$ values of the $\mathrm{S} 1, \mathrm{~S} 2$ and $\mathrm{S} 3$ samples are $0.94 \mathrm{~kJ} \cdot \mathrm{mol}^{-1}, 0.85 \mathrm{~kJ} \cdot \mathrm{mol}^{-1}$ and $0.81 \mathrm{~kJ} \cdot \mathrm{mol}^{-1}$ (all are exothermic), respectively. All these values are within the reference

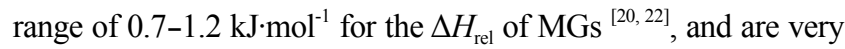
close to the value of $0.89 \mathrm{~kJ} \cdot \mathrm{mol}^{-1}$, which is the $\Delta H_{\text {rel }}$ of the Vit1 copper mold injection cast MG sample with the diameter of 8 $\mathrm{mm}$ and calculated by using the specific heat capacity model ${ }^{[23]}$. By comparing the calculation results, it can be seen that as the 
cooling rate of the alloy melt decreases, the $\Delta H_{\text {rel }}$ of the Vit1 MG samples gradually decreases, indicating that the thermal stability of the amorphous samples gradually increases.

\section{Discussion}

Figure 5 shows the relationships between the melt cooling rates of three groups of Vit1 MG samples and the apparent activation energy of glass transition $E$, the fragility parameter $m$, and the structural relaxation enthalpy $\Delta H_{\text {rel }}$. It can be seen that under the experimental cooling rate conditions of the alloy melt, the $E, m$ and $\Delta H_{\text {rel }}$ values of the Vit1 MG samples are all approximately in line with $\lg R$, that is, they all increase with the increase of the melt cooling rate. It should be noted that when $R<R_{\mathrm{c}}$, the alloy melt cannot solidify into glassy state. However, after the $R$ reaches a certain level, the vitrification process of the alloy melt can be said to be instantaneous, and its structural relaxation time scale in the undercooling stage can be ignored. At this time, the atomic structure of the MG does not change obviously with the further increase of the melt cooling rate $R$. Therefore, it is inferred that the $E, m$ and $\Delta H_{\text {rel }}$ of MGs only change with the melt cooling rate in a certain range.

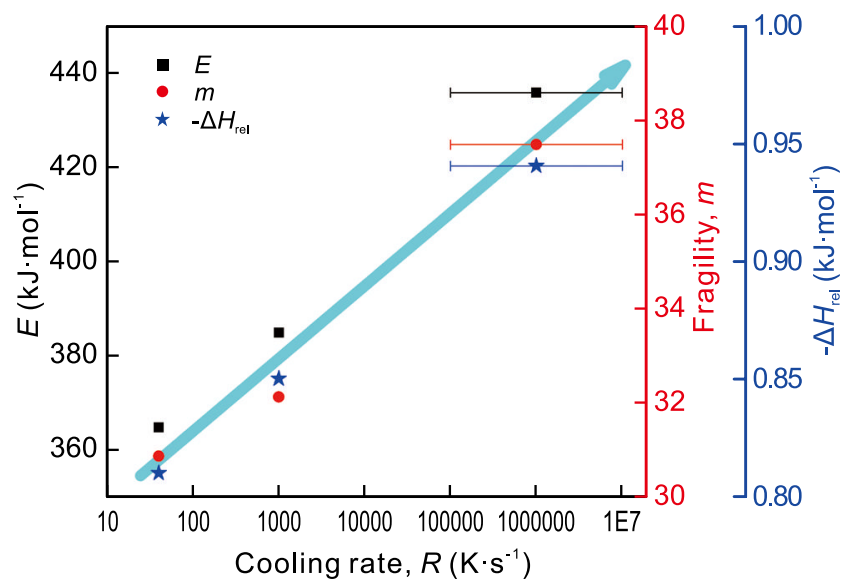

Fig. 5: Relationships between $E, m$ and $\Delta H_{\text {rel }}$ of Vit1 MG sample and cooling rate of alloy melt during sample preparation

In this work, the three groups of Vit1 alloy samples S1$\mathrm{S} 3$ prepared by the melt spinning method and the copper mold injection casting method are all MG, but their obvious differences in glass transition kinetics and structural relaxation enthalpies indicate that their structural characteristics are different at the local atomic scale. For Sample S1 with the highest melt cooling rate, the structural relaxation time of the alloy liquid during the process of vitrification is the shortest, so the atomic structure in this MG sample is closer to that of the alloy melt above the equilibrium melting point, that is, the atoms inside the sample are in a more disordered state, and there is excess free volume in the sample. Conversely, as the cooling rate of the melt decreases, the time for the alloy liquid to undergo the structural relaxation during the process of vitrification gradually becomes longer, that is, both the time scale for the excess free volume in the melt to be annihilated and the time scale for the atoms to transit to an equilibrium state gradually become larger, which will cause the free volume in the two amorphous samples of S2 and S3 to gradually decrease, and the atomic structure in the samples is more orderly and closer to that of the internally balanced alloy liquid with high undercooling. Furthermore, the potential energy of the MG samples is also reduced accordingly. During the heating process, the essential nature of the glass transition of MG is to annihilate the excess free volume through the structural relaxation of atoms in the alloy, so that the atomic structure of the alloy is equilibrated to that of the deep supercooled liquid ${ }^{[24,25]}$. Therefore, the lower the melt cooling rate, that is, the lower the potential energy of the MG sample, the smaller the $\Delta H_{\text {rel }}$ value as its structure is relaxed. At the same time, the thermodynamic barrier potential that it needs to overcome when the glass transition occurs during heating is relatively small, showing a decrease in $T_{\mathrm{g}}$ and $E$. In addition, when it is heated to near $T_{\mathrm{g}}$ and begins to soften, the sensitivity of its viscosity to temperature changes will be less than that of the MG sample with a higher melt cooling rate, showing a reduction in the $m$.

\section{Conclusion}

By using the single roller melt spinning method and the copper mold injection casting method, respectively, three groups of Vit1 MG samples were prepared, and their melt cooling rates spanned at least four orders of magnitude. By using DSC, the glass transition kinetics of the three groups of Vit1 MG samples were quantitatively analyzed at different heating rates. In addition, by using multi-step heating and cooling DSC experiments, the change trends of the structural relaxation enthalpy caused by the melt cooling rate were quantitatively analyzed for the first time. The results show that the melt cooling rate during the solidification of the sample has a significant effect on the glass transition kinetics and structural relaxation enthalpy of the Vit1 MG. Under the experimental conditions, the values of glass transition temperature $T_{\mathrm{g}}$, apparent activation energy $E$ of glass transition, fragility parameter $m$, and structural relaxation enthalpy $\Delta H_{\text {rel }}$ of the Vit1 MG samples all decrease with the decrease of melt cooling rate. This is explained by the theories of free volume change and atomic structure evolution in the structural relaxation of MGs. In addition, it is found that the apparent activation energy, $E$, the glassy fragility parameter, $m$, and structural relaxation enthalpy, $\Delta H_{\text {rel }}$, of the Vit1 MG all have an approximately linear relationship with the natural logarithm of cooling rate, $\lg R$, within the range of the experimental cooling rates.

\section{Acknowledgement}

This work was supported by the National Key Research and Development Program of China (No. 2019YFB2006501). 


\section{References}

[1] Ke $\mathrm{H} \mathrm{B}$, Wen $\mathrm{P}$, Wang $\mathrm{W} \mathrm{H}$. The inquiry of liquids and glass transition by heat capacity. AIP Advances, 2012, 2(4): 041404.

[2] Dyre J C. The glass transition and elastic models of glass-forming liquids. Reviews of Modern Physics, 2006, 78(3): 953-972.

[3] Faupel F, Frank W, Macht M P, et al. Diffusion in metallic glasses and supercooled melts. Reviews of Modern Physics, 2003, 75(1): 237-280

[4] Wen $T Q$, Sun $Y$, Ye B L, et al. Cooling rate dependence of structural order in $\mathrm{Ni}_{62} \mathrm{Nb}_{38}$ metallic glass. Journal of Applied Physics, 2018, 123(4): 045108.

[5] Hayat F, Yin J, Tabassum A, et al. Effects of cooling rate and sub-Tg annealing on $\mathrm{Ni}_{80} \mathrm{P}_{20}$ metallic glass: a molecular dynamic (MD) study. Computational Materials Science, 2020, 179: 109681.

[6] Moynihan C T, Easteal A J, Wilder J, et al. Dependence of the glass transition temperature on heating and cooling rate. The Journal of Physical Chemistry, 1974, 78(26): 2673-2677.

[7] Evenson Z, Gallino I, Busch R. The effect of cooling rates on the apparent fragility of Zr-based bulk metallic glasses. Journal of Applied Physics, 2010, 107(12): 123529.

[8] Sanditov D S, Sydykov B S. Structural relaxation time and cooling rate of a melt in the glass transition region. Russian Journal of Physical Chemistry, 2015, 89(3): 423-428.

[9] Peker A, Johnson W L. A highly processable metallic glass: $\mathrm{Zr}_{41.2} \mathrm{Ti}_{13.8} \mathrm{Ni}_{10.0} \mathrm{Cu}_{12.5} \mathrm{Be}_{22.5}$. Applied Physics Letter, 1993, 63(17): 2342-2344.

[10] Wang X J, Chen X D, Xia T D, et al. Influencing factors and estimation of the cooling rate within an amorphous ribbon. Intermetallics, 2004, 12(10-11): 1233-1237.

[11] Lin X H, Johnson W L. Formation of Ti-Zr-Cu-Ni bulk metallic glasses. Journal of Applied Physics, 1995, 78(11): 6514-6519.

[12] Wang $\mathrm{W} \mathrm{H}$. The nature and properties of amorphous matter Progress in Physics, 2013, 33(5): 177-351. (In Chinese)

[13] Kissinger H E. Reaction kinetics in differential thermal analysis. Analytical Chemistry, 1957, 29(11): 1702-1706.
[14] Li G, Sun L L, Liu R P, et al. Kinetics of glass transition of the $\mathrm{Zr}_{41} \mathrm{Ti}_{14} \mathrm{Cu}_{12.5} \mathrm{Ni}_{10} \mathrm{Be}_{22.5}$ alloy solidified in a drop tube. Chinese Physics Letters, 2002, 19(8): 1148-1151.

[15] Yuan Z Z, Yao L, Bao S L, et al. Effect of melt cooling rate on crystallization and magnetic properties of $\mathrm{Co}_{48} \mathrm{Cr}_{15} \mathrm{Mo}_{14} \mathrm{C}_{15} \mathrm{~B}_{6} \mathrm{Er}_{2}$ bulk metallic glass. Rare Metal Materials \& Engineering, 2009, 38(6): 999-1004. (In Chinese)

[16] Masuhr A, Waniuk T A, Rusch R, et al. Time scales for viscous flow, atomic transport and crystallization in the liquid and supercooled liquid states of $\mathrm{Zr}_{41.2} \mathrm{Ti}_{13.8} \mathrm{Cu}_{12.5} \mathrm{Ni}_{10.0} \mathrm{Be}_{22.5}$. Physical Review Letters, 1999, 82(11): 2290-2293.

[17] Brüning R, Samwer K. Glass transition on long time scales. Physical Review B, 1992, 46(18): 11318-11322.

[18] Perera D N. Compilation of the fragility parameters for several glass-forming metallic alloys. Journal of Physics: Condensed Matter, 1999, 11(19): 3807-3812.

[19] Angell C A. Formation of glasses from liquids and biopolymers. Science, 1995, 267: 1924-1935.

[20] Afonin G V, Mitrofanov Y P, Kobelev N P, et al. Relationship between the enthalpies of structural relaxation, crystallization and melting in metallic glass-forming systems. Scripta Materialia, 2019, 166: 6-9.

[21] Zhang W, Li K L, Xiang Q C, et al. The rejuvenation and relaxation around the glass transition of a Ce-based metallic glass controlled by annealing, quenching and cryogenic treatments. Journal of Non-crystalline Solids, 2020, 548: 120334.

[22] Chen H S. Glass metals. Reports on Progress in Physics, 1980, 43(4): 353-432.

[23] Zhang W, Xiang Q C, Ma C Y, et al. The glass transition during liquid metal solidification exemplified by a $\mathrm{Zr}$-based glassforming alloy: experiments and numerical simulations. AIP Advances, 2020, 10(8): 085225.

[24] Wen P, Tang M B, Pan M X, et al. Calorimetric glass transition in bulk metallic glass forming $\mathrm{Zr}$-Ti-Cu-Ni-Be alloys as a freevolume-related kinetic phenomenon. Physical Review B, 2003, 67(21): 212201.

[25] Busch R, Gallino I. Kinetics, thermodynamics and structure of bulk metallic glass forming liquids. JOM, 2017, 69(11): 2178-2186. 